\title{
FINITE-DIMENSIONAL GLOBAL ATTRACTOR FOR A NONLOCAL PHASE-FIELD SYSTEM
}

\author{
Nota del s.c. MAURIZIO GRASSELLI (*)
}

(Adunanza del 22 marzo 2012)

A mia madre

SunTo. - Analizziamo un sistema di campo di fase in cui l'equazione di bilancio dell'energia è accoppiata linearmente con un'equazione differenziale ordinaria non-lineare e non-locale per il parametro d'ordine $\chi$. Quest'ultima equazione è caratterizzata da una convoluzione spaziale che modella le interazioni a lungo raggio e da un potenziale di configurazione singolare che costringe $\chi$ ad assumere valori nell'intervallo $(-1,1)$. Proviamo che il corrispondente sistema dinamico è dissipativo ovvero possiede un insieme assorbente limitato in un opportuno spazio delle fasi. Stabiliamo quindi l'esistenza di un attrattore globale di dimensione finita.

$* * *$

ABSTRACT. - We analyze a phase-field system where the energy balance equation is linearly coupled with a nonlinear and nonlocal ODE for the order parameter $\chi$. The latter equation is characterized by a space convolution term which models long-range interactions and a singular configuration potential that forces $\chi$ to take values in the interval $(-1,1)$. We prove that the corresponding dynamical system is dissipative, i.e., it has a bounded absorbing set in a suitable phase space. Then we establish the existence of a finite-dimensional global attractor.

(") Dipartimento di Matematica, Politecnico di Milano, Via E. Bonardi 9, 20133 Milano, Italy.

E-mail: maurizio.grasselli@polimi.it 


\section{INTRODUCTION}

Consider a two-phase material (like, for instance, a mixture of ice and water) which occupies a bounded domain in $\Omega \subset \mathbb{R}^{d}, 1 \leq d \leq 3$ and denote by $\vartheta$ its relative temperature with respect to a given (constant) critical one (e.g., the one at which the two phases coexist). A well-known model which accounts for phase changes due to the variation of $\vartheta$ only was proposed and analyzed in [15] (see also [14] and references therein). This is based on the Ginzburg-Landau theory of phase transitions and it assumes as further variable an order parameter (or phase-field) $\chi$ which characterizes, for instance, the most energetic phase of the material (say water, in a water-ice system). Using a phenomenological argument, it is postulated that the evolution of $\chi$ is ruled by a gradient flow of the form

$$
\chi_{t}=-\delta_{\chi} E(\vartheta, \chi),
$$

$\delta_{\chi}$ being the variational derivative with respect to $\chi$ of the free energy $E$ defined by

$$
E(\vartheta, \chi)=\int_{\Omega}\left(\frac{\nu}{2}|\nabla \chi(x)|^{2}+W(\chi(x))-\alpha \vartheta(x) \chi(x)\right) \mathrm{d} x,
$$

where $\nu>0$ and $\alpha \in \mathbb{R}$ are given constants, the latter one being related to the latent heat. Here $W$ is the (density of) potential energy associated with the phase configuration which can be defined either on a finite interval $($ e.g. $(-1,1))$ or on the whole real line. In the first case, $W$ is called singular and the most typical form is the following

$$
W(r)=(1+r) \ln (1+r)+(1-r) \ln (1-r)-\frac{\lambda}{2} r^{2}, \quad r \in(-1,1),
$$

where $\lambda \in \mathbb{R}$. Another important class of potentials are defined on $\mathbb{R}$ and are usually called smooth. A typical example is $W(r)=\left(r^{2}-1\right)^{2}$ which can also be viewed as an approximation of $W$ of $(3)$ on $[-1,1]$.

It is worth observing that the term on the right hand side of (1) may be viewed as a sort of generalized force driving the evolution of $\chi$, that is, the phase transformation. Combining now the balance equation for the internal energy density with (1) we find the evolution system

$$
\begin{aligned}
& (\beta \vartheta+\alpha \chi)_{t}-\gamma \Delta \vartheta=f, \\
& \zeta \chi_{t}-\nu \Delta \chi+W^{\prime}(\chi)-\alpha \vartheta=0,
\end{aligned}
$$


in $\Omega \times(0,+\infty)$, where $f$ represents a given (volumic) heat source and all the constants $\beta, \alpha, \gamma, \zeta, \nu$ are positive. Equations (4)-(5) are also known as Caginalp phase-field system. An important feature of this rather simple model is the fact that its (formal) asymptotic limits are well-known sharp interface problems (see, e.g., [16, 17, 19]). We recall that phasefield systems are diffuse interface models in the sense that the sharp interface separating two different phases, which is usually very difficult to handle theoretically and numerically, is replaced by the level set of a suitable (smooth) order parameter.

From the mathematical viewpoint, system (4)-(5) endowed with initial conditions and various types of boundary conditions has been investigated by many authors (see, e.g., $[10,12,13,20,25,26,27,30,38$, $39,43,44,45,51,56,57,60]$ and references therein), even under more general assumptions (for instance, where $\alpha$ is a function depending on $\chi)$. Besides well-posedness results, an important issue is the longtime behavior of solutions. This behavior is usually non trivial since the set of stationary states is a continuum if the spatial dimension is greater than one. Thus the existence of a Lyapunov functional does not guarantee that a given trajectory converges to a single equilibrium. To prove that the Łojasiewicz-Simon approach has been employed (see, in particular, $[20,25,26,39,60])$. Regarding the global dynamics, system (4)-(5), endowed with appropriate boundary conditions, can be interpreted as a dynamical system in a suitable phase space. This system is dissipative, i.e., there exists a bounded absorbing set in the phase space. In addition, it possesses a finite-dimensional global attractor as well as an exponential attractors (cf., for instance, [10, 12, 13, 20, 38, 44, 45, 51]).

On the other hand, the free energy $E$ can be viewed as an approximation of a nonlocal expression of the following type (see [22] and references therein, cf. also [33, 34, 36, 37] for phase separation models)

$$
\begin{aligned}
& E_{\mathrm{nl}}(\vartheta, \chi) \\
& =\int_{\Omega}\left(\int_{\Omega} k(x-y) \frac{|\chi(x)-\chi(y)|^{2}}{4} \mathrm{~d} y+W(\chi(x))-\alpha \vartheta(x)+\chi(x)\right) \mathrm{d} x,
\end{aligned}
$$

where $k: \mathbb{R}^{d} \rightarrow \mathbb{R}$ is an interaction kernel satisfying $k(x)=k(-x)$ and 
such that

$$
\kappa(x):=\int_{\Omega} k(x-y) \mathrm{d} y
$$

is bounded and nonnegative (see, for instance, [34, Rem. 2.2] for concrete examples). Indeed one can recover (2) by taking

$$
\int_{\Omega} \frac{\nu}{2}|\nabla \chi(x)|^{2} \mathrm{~d} x,
$$

as a first approximation of

$$
\int_{\Omega} \int_{\Omega} k(x-y)|\chi(x)-\chi(y)|^{2} \mathrm{~d} y \mathrm{~d} x,
$$

where $\nu=2 \int_{\Omega} k(y)\left(y_{i}\right)^{2} d y$ is supposed to be independent of coordinate $i$. In concrete examples $k$ is localized in a neighborhood of 0 so that $\nu$ is related to the thickness of the interface between the components. It is interesting to point out that this kind of approximation was already introduced by van der Waals in his celebrated paper [54] and since then it was widely adopted in the mathematical literature on phase transitions. This might be due to the fact that most people are more used to deal with PDE rather than nonlocal operators.

The evolution system which derives from the nonlocal free energy (6) takes the form

$$
\begin{aligned}
& \beta \vartheta_{t}+\alpha \chi_{t}-\Delta \vartheta=f, \\
& \zeta \chi_{t}+\kappa \chi+W^{\prime}(\chi)=\mathcal{J}[\chi]+\alpha \vartheta,
\end{aligned}
$$

in $\Omega \times(0,+\infty)$, where

$$
\mathcal{J}[v](x)=\int_{\Omega} k(x-y) v(y) \mathrm{d} y, \quad x \in \Omega .
$$

Here we have taken $\gamma=1$ for simplicity. The well-posedness for this system was firstly established in [6] through a semigroup approach in 
the case $\Omega=\mathbb{R}$ for a smooth $W$. In a similar setting, existence of traveling waves for small $\alpha$ was proven in [4] (see [3, 5, 7, 8, 11, 21, 32, 59] for various results on the nonlocal Allen-Cahn type equation, i.e., (9) with $\alpha=0$, cf. also the recent monograph [1] for closely related problems). Nucleation simulation by using nonlocal interactions has been studied in [55], while coarsening has been analyzed in [29]. Phase interface dynamics associated with (8)-(9) was analyzed [23, 24] (cf. also references therein and [22]) by formal asymptotics (see also [18] for higher-order approximations and [28] for a related model). The case of bounded multi-dimensional domains was examined in [9] and [31] (cf. also [47, 48, 49, 50, 58] for results on more refined models and [2] for the numerical analysis). Well-posedness issues were discussed in [9] when $\vartheta$ is subject to homogeneous Neumann boundary condition and $W$ is smooth. In addition, some results concerned with the asymptotic behavior were demonstrated (e.g., the existence of a bounded absorbing set in one spatial dimension). In [31], the potential $W$ is smooth as well, but $\vartheta$ is subject to Dirichlet homogeneous. There the main goal was to establish the convergence of a given trajectory to a single stationary state an this fact was proven by means of a generalized version of the Łojasiewicz-Simon inequality, provided that $W$ is real analytic. Note that, also in the nonlocal case, the set of stationary states can be a continuum in dimension greater than one, even though $z \mapsto W^{\prime}(z)+\kappa z$ is invertible (see [31, Introduction], see also [7] for the nonlocal AllenCahn equation). A similar issue is analyzed in the more recent contribution [40], where equation (9) has an additional relaxation term of the form $\varepsilon \chi_{t t}$ and $W$ is a singular potential (though not of type (3)).

However, none of the above results is concerned with the existence of a global attractor even though this is a rather natural feature of phasefield dynamics (cf. above for the local models). In this respect, it is interesting to report the related conclusion of the pioneering paper [6]: Global information, for instance on the existence of an absorbing set and an inertial manifold ..., would also be very useful.

A possible explanation might be that it is not evident how to show some precompactness for the variable $\chi$ (on this point see also [29]). In this note we establish something more than the existence of a bounded absorbing set, namely, the existence of a finite-dimensional global attractor. This is achieved by estimating the differences of two trajecto- 
ries which originated from a suitable bounded absorbing set. Such a difference is controlled by a contractive part depending on the initial data plus a precompact term. For the latter it is essential (but natural) to assume some compactness on the linear nonlocal operator $\mathcal{J}$. For the sake of simplicity, we will take full advantage of the setting developed in [40]. Thus we will suppose that $\vartheta$ satisfies Dirichlet homogeneous boundary condition, while $W(r)$ will be a singular potential defined on $(-1,1)$ which goes to $+\infty$ as $r$ approaches the pure states \pm 1 . This will allow us to easily deduce well-posedness since the model studied in [40] essentially reduces to ours when we take $\varepsilon=0$. The drawback is that one cannot take as initial datum for $\chi$ a function which takes \pm 1 values (i.e., pure phases) in some subset of positive measure. In other (ancient) words corpus inani distinctum, quoniam nec plenum naviter extat nec porro vacuum ([52]). Nonetheless, with some extra-effort, our argument can also be applied to other types of potentials like, for instance, (3) (see Remarks 2.6 and 4.4 below) and to different boundary conditions for $\vartheta$ (e.g., homogeneous Neumann).

The paper is organized as follows. In the next section we discuss well-posedness issues. Section 3 contains a basic uniform in time estimate which is exploited in Section 4 to show that our problem defines a dissipative dynamical system on a suitable phase space. Then we conclude with our main result: the existence of a finite-dimensional global attractor.

\section{WELL-POSEDNESS}

Let us introduce some notation first. Set $H:=L^{2}(\Omega)$ and $V:=H_{0}^{1}(\Omega)$ and denote by $(\cdot, \cdot)$ the scalar product in $H$ and by $\|\cdot\|$ the induced norm. In general, $\|\cdot\|_{X}$ will indicate the norm in a generic real Banach space $X$. Let $V$ be endowed with the norm $\|\cdot\|_{V}:=\|\nabla \cdot\|$. Let us identify $H$ with its topological dual $H^{\prime}$ so that we have the continuous and compact inclusions $V \subset H \subset V^{\prime}$. Moreover, we set $A:=-\Delta$ : $D(A)=H^{2}(\Omega) \cap V \rightarrow H$.

Regarding the potential $W$, we suppose

$$
\begin{aligned}
& W \in C^{2}\left((-1,1) ; \mathbb{R}^{+}\right), \quad \lim _{r \rightarrow 1^{-},-1^{+}} W(r)=+\infty, \\
& \exists \lambda \in \mathbb{R}: \quad W^{\prime \prime}(r) \geq-\lambda, \quad \forall r \in(-1,1) .
\end{aligned}
$$


It is easy to see that (11)-(12) entail

$$
W^{\prime}(r) r \geq W(r)-\frac{\lambda r^{2}}{2}-W(0), \quad \forall r \in(-1,1) .
$$

Moreover, if $v \in L^{\infty}(\Omega)$ is any function such that $W(v) \in L^{\infty}(\Omega)$, then

$$
\begin{aligned}
\exists \delta=\delta\left(W,\|v\|_{L^{\infty}(\Omega)}\right) \in(0,1) \text { such that } & -1+\delta \leq v(x) \\
& \leq 1-\delta \text { for a.e. } x \in \Omega .
\end{aligned}
$$

Consider now $\kappa$ defined by (7) and the nonlocal operator $\mathcal{J}$ (cf. (10)). We require

$$
k \in W^{1,1}\left(\mathbb{R}^{d}\right) .
$$

Observe that assumption (15) entails that $\mathcal{J} \in \mathcal{L}(H ; H)$ is self-adjoint and compact. Also, $\mathcal{J}$ is compact from $L^{\infty}(\Omega)$ to $C(\bar{\Omega})$. Taking some constants equal to one, system (8)-(9) with $\vartheta$ subject to Dirichlet homogeneous boundary condition and initial conditions can now be written as follows

$$
\begin{aligned}
& \vartheta_{t}+\alpha \chi_{t}+A \vartheta=f, \quad \text { a.e. in } \Omega \times(0, T), \\
& \chi_{t}+\kappa \chi+W^{\prime}(\chi)=\mathcal{J}[\chi]+\alpha \vartheta, \quad \text { a.e. in } \Omega \times(0, T), \\
& \vartheta_{\mid t=0}=\vartheta_{0}, \quad \chi_{\mid t=0}=\chi_{0} \quad \text { a.e. in } \Omega,
\end{aligned}
$$

where

$$
\vartheta_{0} \in V
$$

and

$$
f \in H \text {. }
$$

Moreover, we suppose that

$$
\chi_{0} \in L^{\infty}(\Omega) \quad \text { s.t. } \exists \delta_{0} \in(0,1):-1+\delta_{0} \leq \chi_{0} \leq 1-\delta_{0} \quad \text { a.e. in } \Omega \text {, }
$$

where the latter property is equivalent to say that $W\left(\chi_{0}\right) \in L^{\infty}(\Omega)$.

Well-posedness can be proven arguing as in the proof of [40, Thm.2.1] with $\varepsilon=0$ (cf. also [40, Rem.2.7]) 
Theorem 2.1. Let (11)-(12), (15), (19)-(21) hold. Then, for any given $T>0$, there exists one and only one pair $(\vartheta, \chi)$ such that

$$
\begin{gathered}
\vartheta \in L^{2}(0, T ; D(A)) \cap H^{1}(0, T ; H) \cap C^{0}([0, T] ; V), \\
\chi, W(\chi) \in L^{\infty}\left(0, T ; L^{\infty}(\Omega)\right), \quad \chi_{t} \in L^{2}\left(0, T ; L^{\infty}(\Omega)\right),
\end{gathered}
$$

which solves (16)-(18). Moreover, there exists $\delta=\delta\left(W, k_{0}, \kappa, \alpha, f, \delta_{0}\right) \in$ $(0,1)$ such that

$$
-1+\delta \leq \chi(t) \leq 1-\delta \quad \text { a.e. in } \Omega,
$$

for almost any $t \in(0, T)$. Next, given two triplets $\left(\vartheta_{0,1}, \chi_{0,1}\right),\left(\vartheta_{0,2}, \chi_{0,2}\right)$ of initial data satisfying conditions (19)-(21) (the latter w.r.t. possibly different constants $\delta_{0 i}>0, i=1,2$ ) and denoting the corresponding solutions by $\left(\vartheta_{1}, \chi_{1}\right),\left(\vartheta_{2}, \chi_{2}\right)$, respectively, we have the continuous dependence estimate

$$
\begin{gathered}
\left\|\left(\vartheta_{1}-\vartheta_{2}\right)(t)\right\|+\left\|\nabla\left(\vartheta_{1}-\vartheta_{2}\right)\right\|_{L^{2}(0, t ; H)}+\left\|\left(\chi_{1}-\chi_{2}\right)(t)\right\| \\
\leq \Lambda_{0}\left(\left\|\vartheta_{0,1}-\vartheta_{0,2}\right\|+\left\|\chi_{0,1}-\chi_{0,2}\right\|\right), \quad \forall t \in[0, T],
\end{gathered}
$$

where the positive constant $\Lambda_{0}$ depends on $T, \Omega, W, k_{0}, k_{1}, \alpha, f$, and on the initial data (in particular, on $\delta_{0 i}, i=1,2$ ).

Remark 2.2. To establish existence instead of the fixed-point technique used in [40] one can use a vanishing viscosity argument like in [31].

We also have a higher-order control for the temperature difference, namely,

Corollary 2.3. Let the assumptions of Theorem 2.1 hold. In addition to estimate (24) we have

$$
\begin{aligned}
& \left\|\nabla\left(\vartheta_{1}-\vartheta_{2}\right)(t)\right\| \\
& \quad+\left\|A\left(\vartheta_{1}-\vartheta_{2}\right)\right\|_{L^{2}(0, t ; H)} \leq \Lambda_{1}\left(\left\|\vartheta_{0,1}-\vartheta_{0,2}\right\|_{V}+\left\|\chi_{0,1}-\chi_{0,2}\right\|\right),
\end{aligned}
$$

for all $t \in[0, T]$ where $\Lambda_{1}$ is a positive constant similar to $\Lambda_{0}$.

Proof. It suffices to write equation (16) for $\vartheta_{1}-\vartheta_{2}$, multiplying by $A\left(\vartheta_{1}-\vartheta_{2}\right)$ recalling (17) and using (24). 
Remark 2.4. Assumption (15) is satisfied by concrete examples of interaction kernels like the ones mentioned in [34, Rem. 2.2].

Remark 2.5. Assumptions (19) and (20) can be weakened as follows

$$
\vartheta \in D\left(A^{\frac{\rho}{2}}\right), \quad f \in D\left(A^{(\rho-1) / 2}\right),
$$

where $\rho \in\left(\frac{3}{4}, 1\right)$. In this case, we recall that $D\left(A^{\varrho}\right) \subset H^{2 \varrho}(\Omega) \hookrightarrow$ $L^{\infty}(\Omega)$ since the spatial dimension is three at most. In this case estimate (25) becomes

$$
\begin{aligned}
& \left\|A^{\frac{\rho}{2}}\left(\vartheta_{1}-\vartheta_{2}\right)(t)\right\|+\left\|A^{\frac{\rho+1}{2}}\left(\vartheta_{1}-\vartheta_{2}\right)\right\|_{L^{2}(0, t ; H)} \\
& \quad \leq \Lambda_{1}\left(\left\|A^{\frac{\rho}{2}}\left(\vartheta_{0,1}-\vartheta_{0,2}\right)\right\|+\left\|\chi_{0,1}-\chi_{0,2}\right\|\right), \quad \forall t \in[0, T] .
\end{aligned}
$$

Remark 2.6. The second assumption (11) does not allow us to consider the presence of pure phases in the system (see also (21)). Note that, for instance, potentials like (3) should be bounded in $[-1,1]$. This case can still be handled if we deal with the Allen-Cahn equation only and temperature is assumed to be given (see Remark 4.4 below). However, system (8)-(9) with more general potentials require further arguments and have been analyzed elsewhere (see [41]).

\section{A Dissipative ESTIMATE}

Here we establish some uniform in time estimates which are essentially contained in the proof of [40, Thm.2.1] taking $\varepsilon=0$.

Theorem 3.1. Let the assumptions of Theorem 2.1 hold. Then the unique solution $(\vartheta, \chi)$ to (16)-(18) satisfies the following estimate

$$
\|\vartheta(t)\|_{V}^{2}+\|W(\cdot, \chi(t))\|_{L^{\infty}(\Omega)} \leq C_{0}\left(1+c_{\delta_{0}}+\left\|\vartheta_{0}\right\|_{V}^{2}\right) e^{-\varsigma t}+C_{1},
$$

for any $t \geq 0$. Here $\varsigma, C_{0}$ and $C_{1}$ are positive constants which depend on $\Omega, \lambda, W(0), k_{1}, \alpha$ and $f$ at most, while $c_{\delta_{0}}>0$ depends on $\delta_{0}$ and $W$.

Proof. Let us multiply equation (16) by $\vartheta(t)+\xi A \vartheta(t)$ for some given $\xi>0$ to be chosen in the sequel. Integrating over $\Omega$, we get

$$
\frac{\mathrm{d}}{\mathrm{d} t}\left(\frac{1}{2}\|\vartheta\|^{2}+\frac{\xi}{2}\|\nabla \vartheta\|^{2}\right)+\|\nabla \vartheta\|^{2}+\xi\|A \vartheta\|^{2}=\left(f-\alpha \chi_{t}, \vartheta+\xi A \vartheta\right) .
$$


Consider now equation (17). Multiplying it by $\chi_{t}(t)+\eta \chi(t)$, where $\eta>0$ will be fixed in the sequel, and integrating over $\Omega$ we find

$$
\begin{aligned}
\frac{\mathrm{d}}{\mathrm{d} t}( & \left.\frac{\eta}{2}\|\chi\|^{2}+\frac{1}{2}(\kappa \chi, \chi)+(W(\chi), 1)\right)+\left\|\chi_{t}\right\|^{2}+\eta(\kappa \chi, \chi) \\
& +\eta\left(W^{\prime}(\chi), \chi\right)=\left(\mathcal{J}[\chi]+\alpha \vartheta, \chi_{t}+\eta \chi\right) .
\end{aligned}
$$

Adding the two identities we obtain

$$
\begin{aligned}
& \frac{\mathrm{d}}{\mathrm{d} t} \mathcal{E}+\|\nabla \vartheta\|^{2}+\xi\|A \vartheta\|^{2}+\left\|\chi_{t}\right\|^{2}+\eta(\kappa \chi, \chi)+\eta\left(W^{\prime}(\chi), \chi\right) \\
& =(f, \vartheta+\xi A \vartheta)-\xi \alpha\left(\chi_{t}, A \vartheta\right)+\left(\mathcal{J}[\chi], \chi_{t}+\eta \chi\right)+\eta \alpha(\vartheta, \chi),
\end{aligned}
$$

where, for all $t \geq 0$,

$$
\begin{aligned}
\mathcal{E}(t)= & \frac{1}{2}\|\vartheta(t)\|^{2}+\frac{\xi}{2}\|\nabla \vartheta(t)\|^{2}+\frac{\eta}{2}\|\chi(t)\|^{2} \\
& +\frac{1}{2}(\kappa \chi(t), \chi(t))+(W(\chi(t)), 1) .
\end{aligned}
$$

Recalling (13), (15) and the fact that $|\chi| \leq 1$ almost everywhere in $\Omega \times$ $(0, T)$, for any given $T>0$, it is not difficult to choose $\xi$ and $\eta$ such that

$$
\frac{\mathrm{d}}{\mathrm{d} t} \mathcal{E}+c_{1} \mathcal{E}+c_{2}\left(\|A \vartheta\|^{2}+\left\|\chi_{t}\right\|^{2}\right) \leq c_{3}+c_{4}\|f\|^{2},
$$

where $c_{i}, i=1, \ldots, 4$, are positive constants. In particular, $c_{1}$ and $c_{2}$ depend on $\alpha$, while $c_{3}$ depends on $\Omega, \lambda, W(0)$ and $k_{1}$. Here we have also used the Young and Poincaré inequalities.

We now argue as in [40, Proof of Thm.2.1] and we test (17) by $\chi_{t}+\sigma \chi$, for some $\sigma>0$ to be properly selected, but we do not integrate over $\Omega$. We have

$$
\frac{\mathrm{d}}{\mathrm{d} t} \mathcal{G}+\left(\chi_{t}\right)^{2}+\sigma \kappa \chi^{2}+\sigma W^{\prime}(\chi) \chi=(\mathcal{J}[\chi]+\alpha \vartheta)\left(\chi_{t}+\sigma \chi\right),
$$

where $\mathcal{G}(x, t)=\frac{\sigma}{2}(\chi(x, t))^{2}+\frac{1}{2} \kappa(x)(\chi(x, t))^{2}+W(\chi(x, t)), \quad$ a.e. in $\Omega, t \geq 0$. 
Reasoning as before and using Young's inequality, we get

$$
\begin{aligned}
& \frac{\mathrm{d}}{\mathrm{d} t} \mathcal{G}(x, \cdot)+c_{5} \mathcal{G}(x, \cdot)+c_{6}\left(\chi_{t}(x, \cdot)\right)^{2} \\
& \quad \leq c_{7}\left(1+|\vartheta(x, \cdot)|^{2}\right), \quad \text { for a.a. } x \in \Omega,
\end{aligned}
$$

where $c_{i}, i=5, \ldots, 7$ are positive constants depending at most on $\Omega, \lambda$, $W(0), k_{1}$ and $\alpha$. Thanks to the continuous embedding $D(A) \subset L^{\infty}(\Omega)$, we deduce

$$
\frac{\mathrm{d}}{\mathrm{d} t} \mathcal{G}(x, \cdot)+c_{5} \mathcal{G}(x, \cdot)+c_{6}\left(\chi_{t}(x, \cdot)\right)^{2} \leq t c_{8}\left(1+\|A \vartheta\|^{2}\right), \quad \text { for a.a. } x \in \Omega .
$$

If we multiply the above equation by $c_{9}=\frac{c_{2}}{2 c_{8}}$ and we add it to (28), we obtain

$$
\frac{\mathrm{d}}{\mathrm{d} t}\left(\mathcal{E}+c_{9} \mathcal{G}(x, \cdot)\right)+c_{1} \mathcal{E}+c_{9} \mathcal{G}(x, \cdot)+\frac{c_{2}}{2}\|A \vartheta\|^{2}+c_{10}\left\|\chi_{t}\right\|^{2} \leq c_{11}\left(1+\|f\|^{2}\right),
$$

for almost any $x \in \Omega$.

Applying now Gronwall's inequality to (31) we get, for all $t \geq 0$,

$$
\begin{aligned}
\mathcal{E}(t)+c_{9} \mathcal{G}(x, t) \leq & \left(\mathcal{E}(0)+c_{9} \mathcal{G}(x, 0)\right) e^{-\mu t} \\
& +\frac{2 c_{11}}{\mu_{1}}\left(1+\|f\|^{2}\right), \quad \text { for a.a. } x \in \Omega,
\end{aligned}
$$

where $\mu_{1}=\min \left\{1, c_{1}\right\}$, which yields (27).

\section{EXISTENCE OF THE GLOBAL ATTRACTOR}

A consequence of inequality (27) is that $\mathcal{B}(R)=\{u \in V:\|u\| \leq$ $R\}$ for a fixed $R>\sqrt{C_{1}}$ is absorbing for $\vartheta(t)$ as well as for $W(\chi(t))$. Therefore, consider, for instance,

$$
X=\mathcal{B}(R) \times\left\{v \in L^{\infty}(\Omega):|v| \leq 1-\delta_{1}, \text { a.e. in } \Omega\right\},
$$

where $\delta_{1} \in(0,1)$ is such that

$$
\left\{r \in(-1,1): W(r) \leq R^{2}\right\} \subseteq\left[-1+\delta_{1}, 1+\delta_{1}\right] .
$$


If, for each $\left(\vartheta_{0}, \chi_{0}\right) \in X$ and any $t \geq 0$, we define

$$
S(t)\left(\vartheta_{0}, \chi_{0}\right)=(\vartheta(t), \chi(t)),
$$

then, thanks to (27), there exists $t_{0}>0$ such that $S(t) X \subseteq X$ for all $t \geq t_{0}$. If we endow $X$ with the $V \times H$-metric then we obtain a complete (bounded) metric space and $S(t)$ is strongly (Lipschitz) continuous semigroup on $X$ owing to (24) and (25). We now prove the main result of this note, namely, the dynamical system $(X, S(t))$ has a finitedimensional global attractor.

Theorem 4.1. Let the assumptions of Theorem 2.1 hold. In addition, suppose that

$$
\lambda_{0}:=\text { ess } \inf _{x \in \Omega} \kappa(x)-\lambda>0 .
$$

Then $(X, S(t))$ possesses a finite-dimensional connected global attractor.

Proof. Consider $\left(\vartheta_{0 i}, \chi_{0 i}\right) \in X, i=1,2$, set

$$
(\vartheta(t), \chi(t))=\left(\left(\vartheta_{1}-\vartheta_{2}\right)(t),\left(\chi_{1}-\chi_{2}\right)(t)\right)
$$

where $\left(\vartheta_{i}(t), \chi_{i}(t)\right)=S(t)\left(\vartheta_{0 i}, \chi_{0 i}\right)$ for $t \geq t_{0}$, and observe that

$$
\begin{aligned}
& \vartheta_{t}+\alpha \chi_{t}+A \vartheta=0, \quad \text { a.e. in } \Omega \times\left(t_{0},+\infty\right), \\
& \chi_{t}+\kappa \chi+W^{\prime}\left(\chi_{1}(t)\right)-W^{\prime}\left(\chi_{2}(t)\right)=\mathcal{J}[\chi]+\alpha \vartheta, \quad \text { a.e. in } \Omega \times\left(t_{0},+\infty\right) .
\end{aligned}
$$

Let us multiply equation (34) by $A \vartheta(t)$. Integrating over $\Omega$, we get

$$
\frac{1}{2} \frac{\mathrm{d}}{\mathrm{d} t}\|\nabla \vartheta\|^{2}+\|A \vartheta\|^{2}=-\left(\alpha \chi_{t}, A \vartheta\right) .
$$

from which, using the Young and Poincaré inequalities, we derive the estimate

$$
\frac{\mathrm{d}}{\mathrm{d} t}\|\nabla \vartheta\|^{2}+c\|\nabla \vartheta\|^{2} \leq c_{\alpha}\left\|\chi_{t}\right\|^{2} .
$$

and, by comparison in (35), we deduce

$$
\frac{\mathrm{d}}{\mathrm{d} t}\|\nabla \vartheta\|^{2}+c\|\nabla \vartheta\|^{2} \leq c\left(k_{1}, \delta_{1}, \alpha\right)\left(\|\chi\|^{2}+\|\mathcal{J}[\chi]\|^{2}+\|\vartheta\|^{2}\right),
$$


for all $t \geq t_{0}$. On the other hand, multiplying (35) by $\chi(t)$ and integrating over $\Omega$ we find

$$
\frac{1}{2} \frac{\mathrm{d}}{\mathrm{d} t}\|\chi\|^{2}+(\kappa \chi, \chi)+\left(W^{\prime}\left(\chi_{1}\right)-W^{\prime}\left(\chi_{2}\right), \chi\right)=(\mathcal{J}[\chi], \chi)+\alpha(\vartheta, \chi),
$$

and (33) entails

$$
\frac{1}{2} \frac{\mathrm{d}}{\mathrm{d} t}\|\chi\|^{2}+\lambda_{0}\|\chi\|^{2} \leq(\mathcal{J}[\chi], \chi)+\alpha(\vartheta, \chi) .
$$

Then, Young's inequality gives

$$
\frac{1}{2} \frac{\mathrm{d}}{\mathrm{d} t}\|\chi\|^{2}+\frac{\lambda_{0}}{2}\|\chi\|^{2} \leq c_{\lambda_{0}}\|\mathcal{J}[\chi]\|^{2}+c_{\alpha, \lambda_{0}}\|\vartheta\|^{2} .
$$

Since $\mathcal{J}$ is compact and self-adjoint we can find a finite-dimensional projector $\Pi_{\lambda_{0}}$ such that

$$
\|\mathcal{J}[v]\|^{2} \leq \frac{\lambda_{0}}{4 c_{\lambda_{0}}}\|v\|^{2}+\left\|\Pi_{\lambda_{0}}[v]\right\|^{2},
$$

for all $v \in H$. As a consequence we have

$$
\frac{1}{2} \frac{\mathrm{d}}{\mathrm{d} t}\|\chi\|^{2}+\frac{\lambda_{0}}{4}\|\chi\|^{2} \leq c_{\lambda_{0}}\|\Pi[\chi]\|^{2}+c_{\alpha, \lambda_{0}}\|\vartheta\|^{2} .
$$

Adding inequality(36) multiplied by $\mu_{2}=\frac{\lambda_{0}}{8 c\left(k_{1}, \delta_{1}, \alpha\right)}$ to (38) and using (37) yield

$$
\begin{aligned}
& \frac{\mathrm{d}}{\mathrm{d} t}\left(\mu_{2}\|\nabla \vartheta\|^{2}+\|\chi\|^{2}\right) \\
& \quad+c \mu_{2}\|\nabla \vartheta\|^{2}+\frac{\lambda_{0}}{8}\|\chi\|^{2} \leq c\left(\lambda_{0}, k_{1}, \delta_{1}, \alpha\right)\left(\|\Pi[\chi]\|^{2}+\|\vartheta\|^{2}\right) .
\end{aligned}
$$

Therefore, from (39), we deduce

$$
\begin{aligned}
\|\vartheta(t)\|_{V}^{2}+\|\chi(t)\|^{2} \leq & c\left(\lambda_{0}, k_{1}, \delta_{1}, \alpha\right) e^{-\mu_{3}\left(t-t_{0}\right)}\left(\left\|\vartheta\left(t_{0}\right)\right\|_{V}^{2}+\left\|\chi\left(t_{0}\right)\right\|^{2}\right) \\
& +c\left(\lambda_{0}, k_{1}, \delta_{1}, \alpha\right) \int_{t_{0}}^{t}\left(\|\vartheta(\tau)\|^{2}+\left\|\Pi_{\lambda_{0}}[\chi(\tau)]\right\|^{2}\right) d \tau,
\end{aligned}
$$


for all $t \in\left[t_{0}, T\right]$ and any fixed $T>t_{0}$. Here $\mu_{3}$ is a positive constant depending on $\lambda_{0}, k_{1}, \delta_{1}, \alpha$.

We now introduce the following pseudometric in $X$

$$
\begin{aligned}
\mathrm{d}_{T}\left(\left(\vartheta_{01}, \chi_{01}\right),\left(\vartheta_{02}, \chi_{02}\right)\right) \\
\quad=\left(\int_{t_{0}}^{T}\left(\left\|\left(\vartheta_{1}-\vartheta_{2}\right)(\tau)\right\|^{2}+\left\|\Pi_{\lambda_{0}}\left[\left(\chi_{1}-\chi_{2}\right)(\tau)\right]\right\|^{2}\right) d \tau\right)^{1 / 2}
\end{aligned}
$$

and we recall that a pseudometric is (pre)compact in $X$ (with respect to the topology induced by the $X$-metric) if any bounded sequence in $X$ contains a Cauchy subsequence with respect to $\mathrm{d}_{T}$ (see, for instance, [46, Def. 2.57]).

Let us prove that $\mathrm{d}_{T}$ is precompact in $X$. Let $\left\{\left(\vartheta_{0 n}, \chi_{0 n}\right)\right\} \subset X$ ( $X$ is bounded) and set $\left(\vartheta_{n}(t), \chi_{n}(t)\right)=S(t)\left(\vartheta_{0 n}, \chi_{0 n}\right)$. Thanks to (22), we have that $\left\{\vartheta_{n}\right\}$ is bounded in $L^{2}\left(t_{0}, T ; D(A)\right) \cap H^{1}\left(t_{0}, T ; H\right)$. Therefore it contains a subsequence which strongly converges in $L^{2}\left(t_{0}, T, V\right)$. On the other hand, we have that $\left\{\Pi_{\lambda_{0}}\left[\chi_{n}\right]\right\}$ is bounded in $L^{\infty}\left(t_{0}, T ; H\right)$. Also, by comparison in (17), we deduce that $\left\{\left(\chi_{n}\right)_{t}\right\}$ is bounded in $L^{\infty}\left(t_{0}, T ; H\right)$. Therefore $\left\{\left(\Pi_{\lambda_{0}}\left[\chi_{n}\right]\right)_{t}\right\}$ is bounded in $L^{\infty}\left(t_{0}, T ; H\right)$ as well. Then $\left\{\Pi_{\lambda_{0}}\left[\chi_{n}(\cdot)\right]\right\}$ contains a subsequence which strongly converges in $L^{2}\left(t_{0}, T ; H\right)$. Summing up $\left\{\left(\vartheta_{0 n}, \chi_{0 n}\right)\right\}$ contains a Cauchy subsequence with respect to $\mathrm{d}_{T}$.

From (40), we deduce that there exists $t^{*}>t_{0}$ such that

$$
\begin{aligned}
\| S\left(t^{*}\right) & \left(\vartheta_{01}, \chi_{01}\right)-S\left(t^{*}\right)\left(\vartheta_{02}, \chi_{02}\right) \|_{X} \\
\leq & \frac{1}{2}\left\|\left(\vartheta_{01}-\vartheta_{02}, \chi_{01}-\chi_{02}\right)\right\|_{X} \\
& +C\left(\lambda_{0}, k_{1}, \delta_{1}, \alpha\right) \mathrm{d}_{t^{*}}\left(\left(\vartheta_{01}, \chi_{01}\right),\left(\vartheta_{02}, \chi_{02}\right)\right) .
\end{aligned}
$$

Hence $S(t)$ has a (connected) global attractor (see [46, Thm. 2.56, Prop. 2.59]) of finite fractal (i.e., box counting) dimension (cfr. [42, Thm. 2.8.1]).

Remark 4.2. Assumption (33) seems unavoidable when one wants to investigate the long-time behavior of solutions (cf. [9, (A4)] and [31, (1.19)]). In particular, thanks to this assumption, if we take $f \equiv 0$ and 
suppose $W$ real analytic then, on account of [40, Thm. 2.6], we have that the $\omega$-limit set of any pair $\left(\theta_{0}, \chi_{0}\right)$ satisfying (19) and (21) reduces to a singleton $\left\{\left(0, \chi_{\infty}\right)\right\}$, where

$$
\kappa \chi_{\infty}+W^{\prime}\left(\chi_{\infty}\right)=\mathcal{J}\left[\chi_{\infty}\right], \quad \text { a.e. in } \Omega .
$$

Remark 4.3. On account of Remark 2.5, we could take a larger phase space by replacing $V$ with $V_{\rho}=D\left(A^{\frac{\rho}{2}}\right), \rho \in\left(\frac{3}{4}, 1\right)$, in the definition of $\mathcal{B}(R)$ and endowing $X$ with $V_{\rho} \times H$-norm.

Remark 4.4. Consider the following equation

$$
\chi_{t}+\kappa \chi+W^{\prime}(\chi)=\mathcal{J}[\chi]+g,
$$

where $g \in L^{\infty}(\Omega \times(0,+\infty))$. In this case it is possible to show a (uniform) strict separation property even when $W$ is a more general potential like, e.g., (3). However, one should use a comparison argument like, e.g., in [39]. Indeed, it is no longer sufficient to show the global uniform boundedness of $W(\chi)$ (cf. (27)). Then one can define a phase space given by the second component of $X$ (see (32)) and, arguing as above for the $\chi$ component only, prove the existence of a finite-dimensional global attractor.

\section{ACKNOWLEDGMENTS}

The author thanks S. Frigeri, D. Pražák, and G. Schimperna for their helpful remarks. 


\section{REFERENCES}

[1] F. Andreu-Vaillo, J.M. Mazón, J.D. Rossi, J. Julián Toledo-Melero, "Nonlocal diffusion problems", Mathematical Surveys and Monographs, 165. American Mathematical Society, Providence, RI; Real Sociedad Matemática Española, Madrid, 2010.

[2] S. Armstrong, S. Brown, J. Han, Numerical analysis for a nonlocal phase field system, Int. J. Numer. Anal. Model. Ser. B, 1 (2010), 1-19.

[3] P.W. Bates, S. Brown, J. Han, Numerical analysis for a nonlocal Allen-Cabn equation, Int. J. Numer. Anal. Model., 6 (2009), 33-49.

[4] P.W. Bates, F. Chen, Traveling wave solutions for a nonlocal phase-field system, Interfaces Free Bound., 4 (2002), 227-238.

[5] P.W. Bates, F. Chen, Spectral analysis and multidimensional stability of traveling waves for nonlocal Allen-Cahn equation, J. Math. Anal. Appl., 273 (2002), 45-57.

[6] P.W. Bates, F. Chen, J. Wang, Global existence and uniqueness of solutions to a nonlocal phase-field system, in: P.W. Bates, S.-N. Chow, K. Lu and X. Pan, Editors, US-Chinese Conference on Differential Equations and Applications, International Press, Cambridge, MA (1997), 14-21.

[7] P.W. Bates, A. Chmaj, An integrodifferential model for phase transitions: stationary solutions in higher space dimensions, J. Statist. Phys., 95 (1999), 1119-1139.

[8] P.W. Bates, P.C. Fife, X. Ren, X. Wang, Traveling waves in a convolution model for phase transitions, Arch. Rational Mech. Anal., 138 (1997), 105-136.

[9] P.W. Bates, J. Han, G. Zhao, On a nonlocal phase-field system, Nonlinear Anal., 64 (2006), 2251-2278.

[10] P.W. Bates, S. Zheng, Inertial manifolds and inertial sets for the phase-field equations, J. Dynamics Differential Equations, 4 (1992), 375-397.

[11] S.K. Bhowmik, D.B. Duncan, M. Grinfeld, G.J. Lord, Finite to infinite steady state solutions, bifurcations of an integro-differential equation, Discrete Contin. Dyn. Syst. Ser. B, 16 (2011), 57-71.

[12] D. Brochet, X. Chen, D. Hilhorst, Finite dimensional exponential attractor for the phase field model, Appl. Anal., 49 (1993), 197-212.

[13] D. Brochet, D. Hilhorst, Universal attractor and inertial sets for the phase-field model, Appl. Math. Lett., 4 (1991), 59-62.

[14] M. Brokate, J. Sprekels, "Hysteresis and Phase Transitions”, Springer, New York, 1996. 
[15] G. Caginalp, An analysis of a phase field model of a free boundary, Arch. Rational Mech. Anal., 92 (1986), 205-245.

[16] G. Caginalp, Stefan and Hele-Shaw type models as asymptotic limits of the phasefield equations, Phys. Rev. A, 39 (1989), 5887-5896.

[17] G. Caginalp, X. Chen, Convergence to the phase field model to its sharp interface limits, European J. Appl. Math., 9 (1998), 417-445.

[18] G. Caginalp, E. Esenturk, A phase field model with non-local and anisotropic potential, Discrete Contin. Dyn. Syst. Ser. S, 4 (2011) 311-350.

[19] G. Caginalp, P.C. Fife, Dynamics of layered interfaces arising from phase boundaries, SIAM J. Appl. Math., 48 (1988), 506-518.

[20] C. Cavaterra, C.G. Gal, M. Grasselli, A. Miranville, Phase-field systems with nonlinear coupling and dynamic boundary conditions, Nonlinear Anal., 72 (2010), 2375-2399.

[21] X. Chen, Existence, uniqueness and asymptotic stability of traveling waves in nonlocal evolution equations, Adv. Differential Equations, 2 (1997), 125-160.

[22] C.K. Chen, P.C. Fife, Nonlocal models of phase transitions in solids, Adv. Math. Sci. Appl., 10 (2000), 821-849.

[23] X. Chen, G. Caginalp, E. Esenturk, A phase field model with non-local and anisotropic potential, Modelling Simul. Mater. Sci. Eng., 19 (2011) 045006(8).

[24] X. Chen, G. Caginalp, E. Esenturk, Interface conditions for a phase field model with anisotropic and non-local interactions, Arch. Rational Mech. Anal., 202 (2011), 349-372.

[25] L. Cherfils, A. Miranville, Some results on the asymptotic behavior of the Caginalp system with singular potentials, Adv. Math. Sci. Appl., 16 (2007), 107-129.

[26] R. Chill, E. Fašangová, J. Prüss, Convergence to steady states of solutions of the Cabn-Hilliard and Caginalp equations with dynamic boundary conditions, Math. Nachr., 13 (2006), 1448-1462.

[27] A. Damlamian, N. Kenmochi, N. Sato, Subdifferential operator approach to a class of nonlinear systems for Stefan problems with phase relaxation, Nonlinear Anal., 23 (1994), 115-142.

[28] N. Dirr, A Stefan problem with surface tension as the sharp interface limit of a nonlocal system of phase-field type, J. Statist. Phys., 114 (2004), 1085-1113.

[29] D.B. Duncan, M. Grinfeld, I. Stoleriu, Coarsening in an integro-differential model of phase transitions, European J. Appl. Math., 11 (2000), 561-572. 
[30] C.M. Elliott, S. Zheng, Global existence and stability of solutions to the phase-field equations, in "Free boundary problems," Internat. Ser. Numer. Math. 95, 46-58, Birkhäuser Verlag, Basel, 1990.

[31] E. Feireisl, F. Issard-Roch, H. Petzeltová, A non-smooth version of the EojasiewiczSimon theorem with applications to non-local phase-field systems, J. Differential Equations, 199 (2004), 1-21.

[32] P.C. Fife, Well-posedness issues for models of phase transitions with weak interaction, Nonlinearity, 14 (2001), 221-238.

[33] H. Gajewski, On a nonlocal model of non-isothermal phase separation, Adv. Math. Sci. Appl., 12 (2002), 569-586.

[34] H. Gajewski, K. Zacharias, On a nonlocal phase separation model, J. Math. Anal. Appl., 286 (2003), 11-31.

[35] C.G. Gal, M. Grasselli, A. Miranville, Robust exponential attractors for singularly perturbed phase-field equations with dynamic boundary conditions, NoDEA Nonlinear Differential Equations Appl., 15 (2008), 535-556.

[36] G. Giacomin, J.L. Lebowitz, Phase segregation dynamics in particle systems with long range interactions. I. Macroscopic limits, J. Statist. Phys., 87 (1997), 37-61.

[37] G. Giacomin, J.L. Lebowitz, Phase segregation dynamics in particle systems with long range interactions. II. Interface motion, SIAM J. Appl. Math., 58 (1998), $1707-1729$.

[38] M. Grasselli, A. Miranville, G. Schimperna, The Caginalp phase-field system with coupled dynamic boundary conditions and singular potentials, Discrete Contin. Dyn. Syst., 28 (2010), 67-98.

[39] M. Grasselli, H. Petzeltová, G. Schimperna, Long time behavior of solutions to the Caginalp system with singular potential, Z. Anal. Anwendungen, 25 (2006), $51-72$.

[40] M. Grasselli, H. Petzeltová, G. Schimperna, A nonlocal phase-field system with inertial term, Quart. Appl. Math., 65 (2007), 451-469.

[41] M. Grasselli, G. Schimperna, Nonlocal phase-field systems with general potentials, Discrete Contin. Dyn. Syst. Ser. A., 33 (2013), 5089-5106.

[42] J.K. Hale, "Asymptotic behaviour of dissipative systems", Amer. Math. Soc., Providence, RI, 1988.

[43] A. Jiménez-Casas, A. Rodríguez-Bernal, Asymptotic behaviour for a phase field model in higher order Sobolev spaces, Rev. Mat. Complut., 15 (2002), 213-248. 
[44] V.K. Kalantarov, On the minimal global attractor of a system of phase field equations, (Russian), Zap. Nauchn. Sem. Leningrad. Otdel. Mat. Inst. Steklov. (LOMI) 188 (1991), Kraev. Zadachi Mat. Fiz. i Smezh. Voprosy Teor. Funktsii. 22, 70-86, 186 [translation in J. Math. Sci., 70 (1994), 1767-1777].

[45] O.V. Kapustyan, An attractor of a semiflow generated by a system of phase-field equations without uniqueness of the solution (Ukrainian), Ukrain. Mat. Zh., 51 (1999), 1006-1009 [Translation in Ukrainian Math. J., 51 (1999), 1135-1139 (2000)].

[46] N.J. Koksch, A.J. Milani, “An introduction to semiflows", Chapman \& Hall/CRC, Boca Raton, FL, 2005.

[47] P. Krejćí, J. Sprekels, Nonlocal phase-field models for non-isothermal phase transitions and hysteresis, Adv. Math. Sci. Appl., 14 (2004), 593-612.

[48] P. Krejčí, J. Sprekels, Long time behavior of a singular phase transition model, Discrete Contin. Dyn. Syst., 15 (2006), 1119-1135.

[49] P. Krejćí, E. Rocca, J. Sprekels, Nonlocal temperature-dependent phase-field models for non-isothermal phase transitions, J. Lond. Math. Soc. (2), 76 (2007), 197210 .

[50] P. Krejčí, E. Rocca, J. Sprekels, A nonlocal phase-field model with nonconstant specific heat, Interfaces Free Bound., 9 (2007), 285-306.

[51] Ph. Laurençot, Long-time behaviour for a model of phase-field type, Proc. Roy. Soc. Edinburgh Sect. A, 126 (1996), 167-185.

[52] Titus Lucretius Carus, “De Rerum Natura”, Liber I.

[53] A. Miranville, S. Zelik, Robust exponential attractors for singularly perturbed phase-field type equations, Electron. J. Differential Equations, 63 (2002), 1-28.

[54] J.S. Rowlinson, Translation of J.D. van der Waals, The thermodynamic theory of capillarity under the bypothesis of a continuous variation of density, J. Statist. Phys., 20 (1979), 197-244.

[55] A. Roy, J.M. Rickman, J.D. Gunton, K.R. Elder, Simulation study of nucleation in a phase-field model with nonlocal interactions, Phys. Rev. E, 57 (1998), 2610(8).

[56] N. Sato, T. Aiki, Phase field equations with constraints under nonlinear dynamic boundary conditions, Commun. Appl. Anal., 5 (2001), 215-234.

[57] G. Schimperna, Abstract approach to evolution equations of phase field type and applications, J. Differential Equations, 164 (2000), 395-430. 
[58] J. Sprekels, S. Zheng, Global existence and asymptotic bebaviour for a nonlocal phase-field model for non-isothermal phase transitions, J. Math. Anal. Appl., 279 (2003), 97-110.

[59] X. Wang, Metastability and stability of patterns in a convolution model for phase transitions, J. Differential Equations, 183 (2002), 434-461.

[60] Z. Zhang, Asymptotic behavior of solutions to the phase-field equations with Neumann boundary conditions, Commun. Pure Appl. Anal., 4 (2005), 683-693. 